\title{
Reversible Lennox-Gastaut Syndrome: an Exemplary Case of Curative Focal Heterotopia Resection
}

Jessica R Fesler ${ }^{1}$, Saad Kanaan', Timothy B Mapstone ${ }^{2}$ and Yu-Tze Ng $^{3 *}$

${ }^{1}$ Department of Neurology, University of Oklahoma, Oklahoma City, Oklahoma

${ }^{2}$ Department of Neurosurgery, University of Oklahoma, Oklahoma City, Oklahoma

${ }^{3}$ Department of Pediatrics, Baylor College of Medicine, Children's Hospital of San Antonio, San Antonio, Texas

\begin{abstract}
Pediatric epilepsy patients with generalized or multifocal abnormalities on electroencephalogram and developmental delay have not classically been considered surgical candidates, even if neuroimaging revealed a lesion. A new paradigm advocates for early focal epilepsy surgery, despite non-localizing EEG abnormalities. This is an instructive case of curative focal subcortical heterotopia resection in a six-year-old boy with refractory, symptomatic generalized epilepsy who fit criteria for Lennox-Gastaut syndrome. Post-surgery, he had normalization of his EEG, resolution of behavioral problems and developmental delay, and no seizure recurrence off all antiepileptic medications. Literature is reviewed in the context of this demonstrative case supporting that generalized electrographic and clinical expression of focal pathology may occur beyond infancy and the principle of secondary epileptogenesis. Epileptic encephalopathy may be reversible with resection of a focal lesion and there is potential for cure if patients are given early surgical consideration.
\end{abstract}

\section{Highlights}

1. Non-localizing clinical semiology or EEG can be misleading.

2. Epileptic encephalopathy can be reversible with focal respective surgery.

3. The best resection is the minimum to stop seizures but preserve eloquent cortex.

Keywords: Secondary epileptogenesis; Epileptic encephalopathy; Sub-cortical heterotopia; Intractable epilepsy; Epilepsy surgery

\section{Introduction}

Epilepsy patients with generalized or multifocal electroencephalogram (EEG) abnormalities and developmental delay were not classically considered surgical candidates, even if neuroimaging revealed a focal lesion. A recent paradigm shift advocates expedited surgical resection of focal lesions despite non-localizing EEG due to expanding knowledge and experience. Current literature reflects this shift, especially in infantile spasms and gelastic epilepsy symptomatic to hypothalamic hamartoma, but it is extrapolating as an over-arching principle in any focal pathology with generalized EEG dysfunction [1-6].

Surgical management is still underutilized despite distribution of Class 1 evidence for effectiveness in refractory temporal lobe epilepsy and Clinical Practice Guidelines [7]. This is even more probable when EEG and magnetic resonance imaging (MRI) are discordant. Presumably there are patients, especially with developmental delay and epileptic encephalopathy, who are potentially being overlooked for epilepsy surgery, when current data suggests they should not be. This is an instructive case of focal heterotopia resection with gradual but complete resolution of seizures and delay, which exemplifies why early surgical consideration is imperative.

\section{Case Summary}

A six-year-old boy with an isolated generalized seizure with fever at age one began having complex partial seizures with occasional secondary generalization and myoclonic jerks at age four. He developed multiple seizure types which became refractory including complex partial, myoclonic, absence, generalized tonic-clonic, tonic, and atonic, as well as diffuse electrographic abnormalities and developmental delay. MRI showed a small left paraventricular subcortical heterotopia (Figure 1)
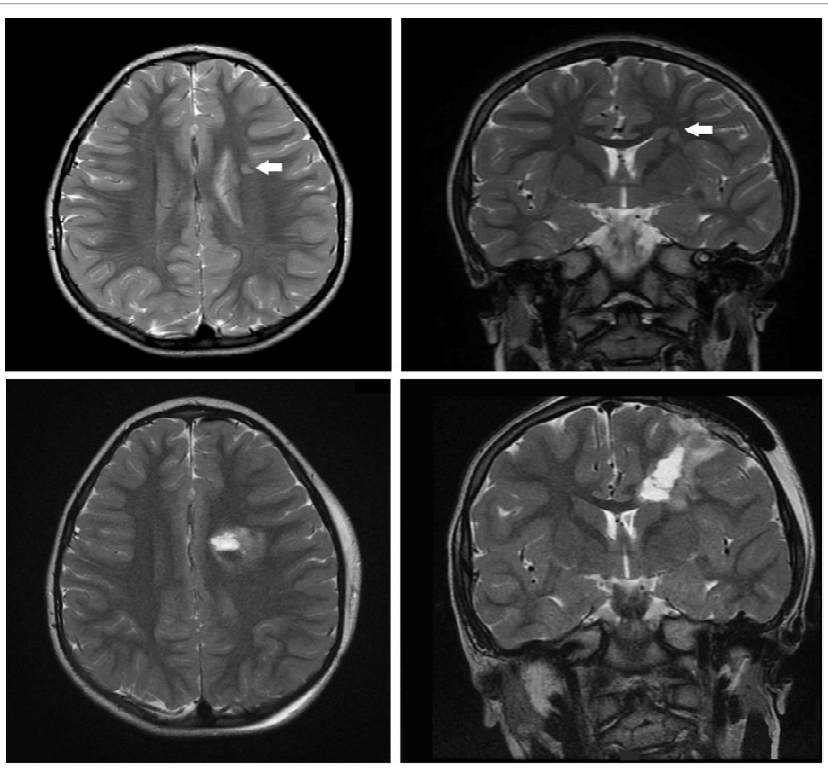

Figure 1: Pre-operative MRI brain T2 axial and coronal images with arrows designating left frontal cortex heterotopic grey matter within the sub ependymal white matter of the left lateral ventricle and corona radiata and immediate post-operative MRI brain T2 axial and coronal images showing left frontal craniotomy with resection of heterotopia.

*Corresponding author: Yu-Tze Ng, Gary Dudley and Charlie Amato Endowed Chair in Pediatric Neurology, Division of Neurology, Children's Hospital of San Antonio, Department of Pediatrics, Baylor College of Medicine, $315 \mathrm{~N}$ San Saba \#1003, San Antonio, TX 78207, USA, Tel: (210)704-8868; Fax: (210)704-4527; E-mail: ytng@bcm.edu

Received August 01, 2015; Accepted September 15, 2015; Published September 22, 2015

Citation: Fesler JR, Kanaan S, Mapstone TB, Ng YT (2015) Reversible LennoxGastaut Syndrome: an Exemplary Case of Curative Focal Heterotopia Resection J Neurol Neurophysiol 6: 315. doi:10.4172/2155-9562.1000315

Copyright: @ 2015 Fesler JR, et al. This is an open-access article distributed unde the terms of the Creative Commons Attribution License, which permits unrestricted use, distribution, and reproduction in any medium, provided the original author and source are credited. 
Citation: Fesler JR, Kanaan S, Mapstone TB, Ng YT (2015) Reversible Lennox-Gastaut Syndrome: an Exemplary Case of Curative Focal Heterotopia Resection. J Neurol Neurophysiol 6: 315. doi:10.4172/2155-9562.1000315

with corresponding decreased uptake on interictal proton emission tomography (18F-FDG PET). Seizures persisted despite adequate medication trials including levetiracetam, pyridoxine, zonisamide, valproic acid, clobazam, topiramate, and clonazepam. He was averaging two atonic and 15 generalized tonic-clonic seizures daily on maximized doses of three antiepileptics (levetiracetam $65 \mathrm{mg} / \mathrm{kg} /$ day, topiramate $15 \mathrm{mg} / \mathrm{kg} /$ day, and clobazam $2 \mathrm{mg} / \mathrm{kg} /$ day). He developed epileptic encephalopathy with verbal delay, severe behavioral issues including explosive anger outbursts, hyperactivity, and poor attention, which precluded enrollment in daycare. Prolonged EEG monitoring showed a generalized pattern of 1.5 to $2.5 \mathrm{~Hz}$ polyspike-and-slow waves, diffuse background slowing, multiple subclinical and subtle generalized seizures without focality, and generalized paroxysmal fast activity during sleep, all consistent with a classic Lennox-Gastaut syndrome picture (Figure 2).
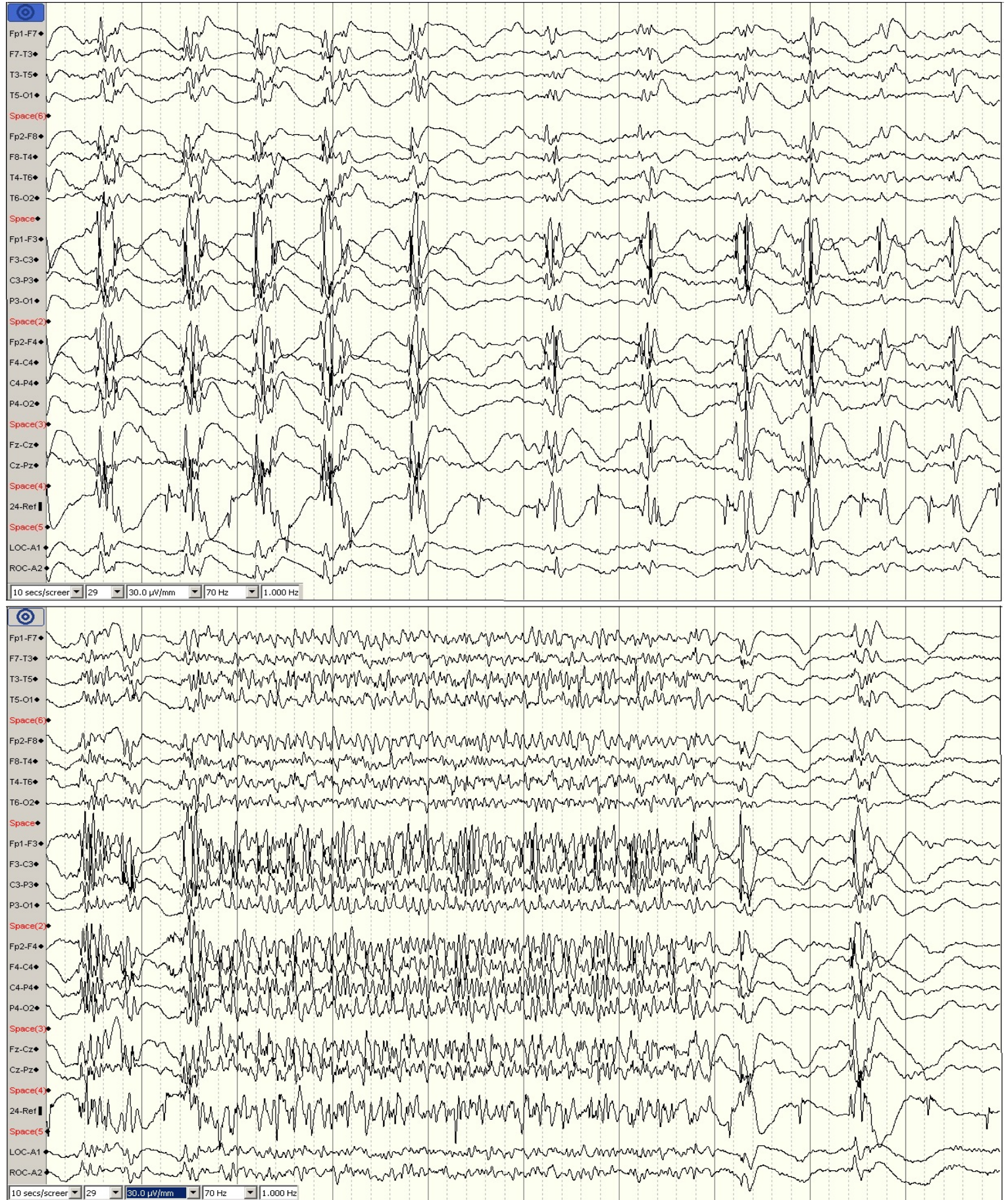

Figure 2: Pre-operative EEG sample showing abundant to near-continuous slow $(1.5-2.5 \mathrm{~Hz})$ generalized (poly) spike-and-slow wave discharges as well as generalized paroxysmal fast activity during sleep. 
Citation: Fesler JR, Kanaan S, Mapstone TB, Ng YT (2015) Reversible Lennox-Gastaut Syndrome: an Exemplary Case of Curative Focal Heterotopia Resection. J Neurol Neurophysiol 6: 315. doi:10.4172/2155-9562.1000315

His intractable epilepsy was presumed symptomatic to the grey matter heterotopia. Surgical resection was agreed upon without further invasive stage II monitoring. He underwent left frontal craniotomy with focal resection of the pathologically confirmed heterotopia before turning seven (Figure 1). Surgery was complicated by mild transient right arm weakness, undetectable after two months. Seizure frequency improved immediately, but he continued to have two complex partial seizures per day. By two months post-resection, he had rare tonic seizures at night and by three months became seizure free. He remained so, even after all antiepileptic medications were tapered off at one year post-resection. Furthermore, his behavior, learning, and speech improved remarkably following surgery, and he was able to attend first grade classes on target with his peers without special education requirement. Electrographic changes paralleled this improvement. EEG was unchanged immediately post-surgery with reduction of the epileptiform discharges at one month and normalization of the EEG by one year (Figure 3). This child, who previously met criteria for LennoxGastaut syndrome, is essentially normal and remains seizure free off medications (over two years to date)!

\section{Discussion}

This patient met criteria for Lennox-Gastaut syndrome, a triad of epileptic encephalopathy with progressive cognitive impairment, behavior disturbance, multiple seizure types, and an EEG with bilaterally synchronous and slow generalized spike-and-wave abnormality [8]. Most patients have non-localizing workup but only $25 \%$ are deemed cryptogenic with no clear lesion on imaging [8].

Diffuse electrographic expression is likely linked to the interaction between the lesion and the developing brain. That a focal lesion in infancy can cause diffuse electrographic abnormality (hypsarrhythmia) and generalized seizure activity (infantile spasms) is well established [1]. Some propose a spectrum exists wherein younger brain manifests hypsarrhythmia, but as the brain matures the same process is evidenced as diffuse slowing and generalized epileptiform discharges [2]. Likewise, the epileptic encephalopathies may lie on a continuum with a predictable pattern of evolution based on age [12]. Up to $40 \%$ of patients with EEG findings consistent with Lennox-Gastaut evolve from infantile spasms with electrographic hypsarrhythmia [13]. However, it is unclear why generalized and multifocal EEG abnormalities do not develop in all children with congenital epileptogenic lesions. Epilepsy surgery may be successful with any congenital brain lesion, despite abundant generalized or bilateral epileptiform discharges on EEG, though data is more limited [3,4]. A new paradigm is forming and within its constructs, generalized and multifocal EEG does not preclude epilepsy surgery in any patient, but especially if there is a known focal lesion on neuroimaging, because a focal epileptogenic process can be masked by generalized EEG abnormalities [4].

The discordant electrical abnormalities, likely secondary phenomena, gradually resolve after resection of the primary pathology. Relevant proposed theories for this process include secondary epileptogenesis, kindling, and maladaptive neural plasticity. The definition of secondary epileptogenesis is debated but generally refers to the creation of separate discharging epileptogenic foci independently capable of generating clinical seizures within the synaptic networks of the original lesion [14]. Kindling is a similar concept referring to experiments in which seizures are evoked by repeated administration of a sub convulsive stimulus that eventually spreads to become secondarily generalized and then spontaneous, leading to changes in plasticity, a permanent state of hyper excitability, and eventually structural and functional changes. Maladaptive neural plasticity refers to the synaptic

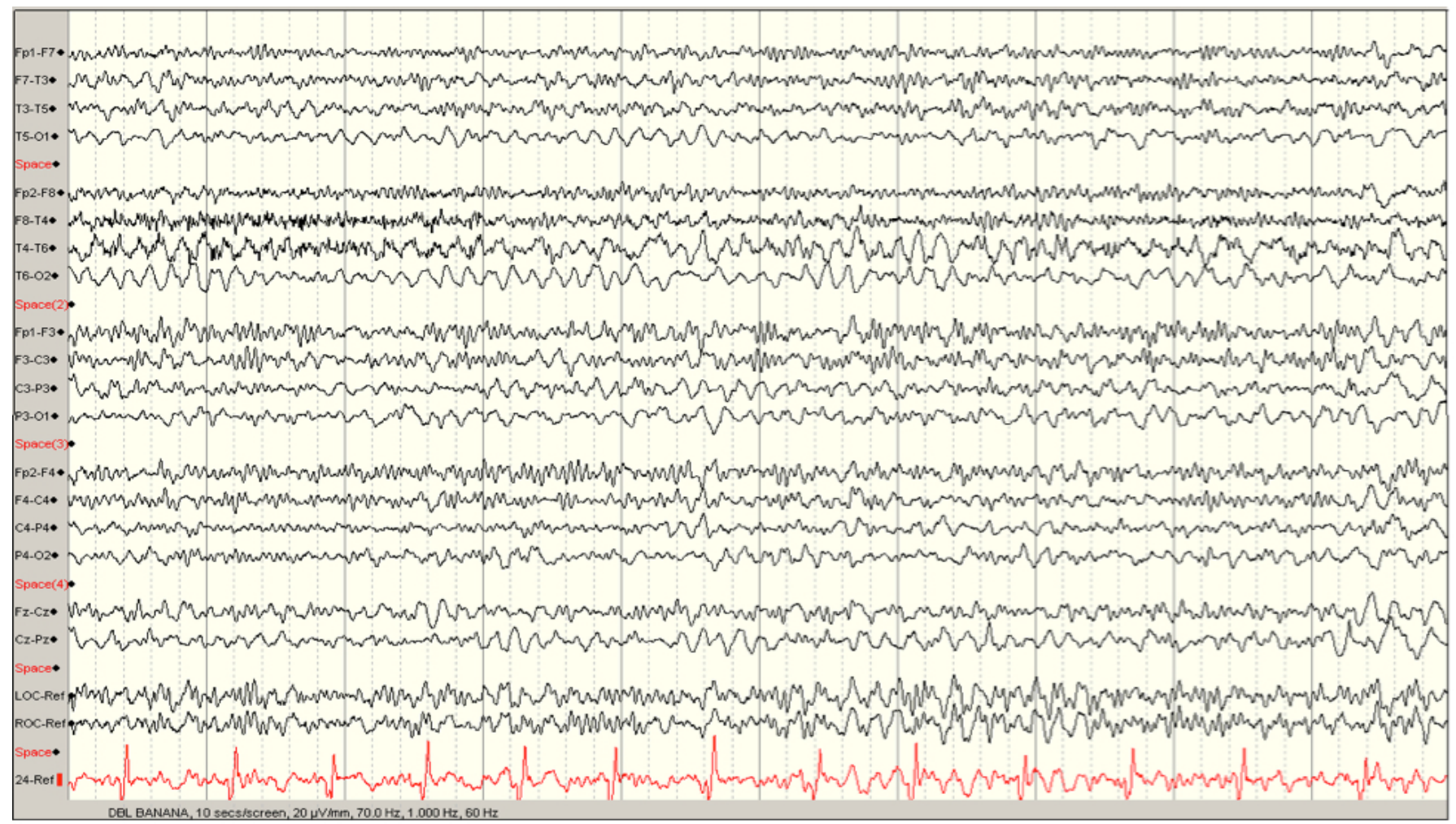

Figure 3: Normal EEG one year post surgical resection. 
Citation: Fesler JR, Kanaan S, Mapstone TB, Ng YT (2015) Reversible Lennox-Gastaut Syndrome: an Exemplary Case of Curative Focal Heterotopia Resection. J Neurol Neurophysiol 6: 315. doi:10.4172/2155-9562.1000315

network dysfunction in the developing brain, causing the unusual EEG manifestations of focal epileptogenic lesions in children [4].

The epileptic encephalopathy is a manifestation of this disturbed circuitry from the interaction of the primary lesion and the developing brain [13]. The effect of frequent early seizures on cognitive potential is significant to clinical outcome. The later the age of onset of encephalopathy the better patients do cognitively, having already undergone certain critical stages of brain development [13]. One study showed the duration of epilepsy influences the ability of a child to "catch up", with shorter intervals leading to more post-operative longterm gains [15]. Fortunately, generalized electrical changes usually precede clinical manifestations of progressive epileptogenesis, giving the potential for quick action [14]. Early surgery reduces the cognitive and behavioral sequelae of chronic epilepsy, likely a key element to this child's remarkable recovery.

The general concept that seizures beget seizures holds true. It is clear that recurrent seizures have a continuous and progressive effect, which is initially reversible but can become irreversible [14]. If surgery is delayed, secondary foci become fully autonomous, unaffected by removal of the primary lesion [6]. Unfortunately, there is no clinical measure or consistent time of transition to this stage of irreversibility. Furthermore, there is dissociation between imaging pathology and epileptogenicity, making effective surgery planning difficult. The lesion seen on imaging is often less epileptic than the normal-appearing borders. Along the same lines as "the correct antiepileptic dose is the lowest that keeps one free of seizures", the correct amount of resected brain is the least amount that cures one's epilepsy while maximally preserving eloquent cortex. However, this is often elusive, impossible to ascertain, and a topic of debate. This case was initially planned as a broader wedge resection rather than the discrete surgery eventually decided upon.

\section{Conclusion}

Many epilepsy patients have generalized or bilaterally independent epileptiform discharges, and a non-focal EEG would likely have precluded surgical consideration until recently. However, as discussed in detail here, generalized expression of focal pathology occurs beyond infancy. Symptomatic generalized epilepsy and resultant epileptic encephalopathy can be reversible with focal resection. Although not always the reality, the best strategy is early resection to stop deleterious effects on the developing brain and halt the epileptogenic network changes before they become irreversible.

\section{Acknowledgements}

This work was done at University of Oklahoma Health Sciences Center.

\section{References}

1. Chugani HT, Shields WD, Shewmon DA, Olson DM, Phelps ME, et al. (1990) Infantile spasms: I. PET identifies focal cortical dysgenesis in cryptogenic cases for surgical treatment. Ann Neurol 27: 406-413.

2. Lee YJ, Kang HC, Lee JS, Kim SH, Kim DS, et al. (2010) Resective pediatric epilepsy surgery in Lennox-Gastaut syndrome. Pediatrics 125: e58-66.
3. Wyllie E, Lachhwani DK, Gupta A, Chirla A, Cosmo G, et al. (2007) Successful surgery for epilepsy due to early brain lesions despite generalized EEG findings. Neurology 69: 389-397.

4. Gupta A, Chirla A, Wyllie E (2007) Pediatric Epilepsy Surgery in Focal Lesions and Generalized Electroencephalogram Abnormalities. Pediatr Neurol 37: 8-15.

5. Troester M, Haine-Schlagel R, Ng YT, Chapman K, Chung S, et al. (2011) EEG and video-EEG seizure monitoring has limited utility in patients with hypothalamic hamartoma and epilepsy. Epilepsia 52: 1137-1143.

6. Scholly J, Valenti MP, Staack AM (2013) Hypothalamic hamartoma: is the epileptogenic zone always hypothalamic? Arguments for independent (third stage) secondary epileptogenesis. Epilepsia 54:123-128.

7. Haneef Z, Stern J, Dewar S, Engel J (2010) Referral pattern for epilepsy surgery after evidence-based recommendations: a retrospective study. Neurology 75 699-704.

8. VanStraten AF, Ng YT (2012) Update on the management of Lennox-Gastaut syndrome. Pediatr Neurol 47: 153-161.

9. Pati S, Deep A, Troester MM, Kossoff EH, Ng YT (2013) Lennox-Gastaut syndrome symptomatic to hypothalamic hamartoma: evolution and long-term outcome following surgery. Pediatr Neurol 49: 25-30.

10. Berkovic SF, Kuzniecky RI, Andermann F (1997) Human epileptogenesis and hypothalamic hamartomas: new lessons from an experiment of nature. Epilepsia 38: 1-3.

11. Freeman JL, Harvey AS, Rosenfeld JV, Wrennall JA, Bailey CA, et al. (2003) Generalized epilepsy in hypothalamic hamartoma: evolution and postoperative resolution. Neurology 60: 762-767.

12. Donat JF (1992) The age-dependent epileptic encephalopathies. J Child Neurol 7: 7-21.

13. Goldsmith IL, Zupanc ML, Buchhalter JR (2000) Long-term seizure outcome in 74 patients with Lennox-Gastaut syndrome: effects of incorporating MRI head imaging in defining the cryptogenic subgroup. Epilepsia 41: 395-399.

14. Morrell F (1989) Varieties of human secondary epileptogenesis. J Clin Neurophysiol 6: 227-275.

15. Freitag $\mathrm{H}$, Tuxhorn I (2005) Cognitive function in preschool children after epilepsy surgery: rationale for early intervention. Epilepsia 46: 561-567. 